\title{
Suspeita de diabetes infanto-juvenil: delineando o contexto familiar e social das crianças e adolescentes atendidas pelo SAMU
}

\author{
Suspected child and adolescent diabetes: outlining the family and social context of the \\ children and adolescents served by EMS
}

Sospecha de diabetes infantil y adolescente: descripción del contexto familiar y social de los niños y adolescentes atendidos por ASMU

Morgana Georgia Fernandes Silva1 ${ }^{1}$ Bianca Machado Cruz Shibukawa ${ }^{1 *}$, Maria de Fátima Garcia Lopes Merino ${ }^{1}$, Gabrieli Patricio Rissi ${ }^{1}$, Vanessa Paula Lanjoni ${ }^{1}$, Roberta Tognollo Borotta Uema ${ }^{1}$, Larissa Carolina Segantini Felipin ${ }^{1}$, Maycon Hoffmann Cheffer ${ }^{1}$, Vanessa Denardi Antoniassi Baldissera ${ }^{1}$, leda Harumi Higarashi'.

\section{RESUMO}

Objetivo: Descrever a configuração familiar e o apoio social de famílias de crianças e adolescentes com suspeita de Diabetes Mellitus tipo 1 atendidos pelo Sistema de Atendimento Móvel de Urgência (SAMU). Métodos: Estudo descritivo e qualitativo, cuja seleção dos sujeitos se deu via banco de dados secundários do SAMU do Noroeste do Paraná no primeiro semestre de 2015. Participaram do estudo três adolescentes com alteração de glicemia no momento do atendimento e que tivesse contato telefônico, uma mãe e um pai. Realizaram-se entrevistas para a construção de genograma e ecomapa, os quais foram analisados de forma descritiva. Resultados: A maioria das famílias são nucleares (pai, mãe e filhos), possuem casa própria e são atendidas pela equipe da atenção básica de saúde. Os vínculos de apoio mais citados pelas participantes foram família extensa, igreja, trabalho, vizinhos e amigos. A relação com a unidade básica de saúde foi considerada por muitas, como uma relação frágil. Conclusão: Há a necessidade do fortalecimento do vínculo entre os adolescentes e familiares com a unidade básica de saúde, de modo que haja confiança para a resolutividade de problemas de saúde, deixando o atendimento pelo Serviço de Atendimento Móvel de Urgências, apenas casos de reais necessidades.

Palavras-chave: Diabetes Mellitus tipo 1, Serviços médicos de emergência, Saúde do adolescente.

\begin{abstract}
Objective: To describe the family configuration and social support of families of children and adolescents with suspected type 1 Diabetes Mellitus treated by the Mobile Emergency Care System (SAMU). Methods: Descriptive and qualitative study, whose selection of subjects took place via the secondary database of SAMU in the Northwest of Paraná in the first semester of 2015. Three adolescents with blood glucose alteration at the time of the attendance and who had telephone contact, participated in the study. mom and dad. Interviews were carried out to construct a genogram and ecomap, which were analyzed descriptively. Results: Most families are nuclear (father, mother and children), have their own home and are served by the primary health care team. The support bonds most cited by the participants were extended family, church, work, neighbors and friends. The relationship with the basic health unit was considered by many to be a fragile one. Conclusion: There is a need to strengthen the bond between adolescents and family members with the basic health unit, so that there is confidence in resolving health problems, leaving care by the Mobile Emergency Service, only cases of real needs.
\end{abstract}

Key words: Diabetes Mellitus type 1, Emergency medical services, Adolescent health.

1 Universidade Estadual de Maringá (UEM), Maringá - PR. *E-mail: bih.cruuz@gmail.com 


\section{RESUMEN}

Objetivo: Describir la configuración familiar y el apoyo social de las familias de niños y adolescentes con sospecha de Diabetes Mellitus tipo 1 atendidos por el Sistema Móvil de Atención de Emergencias (SAMU). Métodos: Estudio descriptivo y cualitativo, cuya selección de sujetos se realizó a través de la base de datos secundaria del SAMU en el Noroeste de Paraná en el primer semestre de 2015. Participaron del estudio tres adolescentes con alteración de la glucemia al momento de la asistencia y que tenían contacto telefónico. mamá y papá. Se realizaron entrevistas para la construcción de un genograma y un ecomap, que se analizaron de forma descriptiva. Resultados: La mayoría de las familias son nucleares (padre, madre e hijos), tienen casa propia y son atendidas por el equipo de atención primaria de salud. Los vínculos de apoyo más citados por los participantes fueron la familia extendida, la iglesia, el trabajo, los vecinos y los amigos. Muchos consideraron que la relación con la unidad básica de salud era frágil. Conclusión: Existe la necesidad de fortalecer el vínculo entre adolescentes y familiares con la unidad básica de salud, para que exista confianza en la resolución de problemas de salud, dejando la atención por el Servicio de Emergencia Móvil, solo casos de necesidades reales.

Palabras clave: Diabetes Mellitus tipo 1, Servicios médicos de urgencia, Salud del adolescente.

\section{INTRODUÇÃO}

O Diabetes Mellitus tipo 1 (DM1) ocorre principalmente em crianças e adolescentes, sendo raro em adultos. Atualmente há um crescente aumento de doença em todo o mundo, estima-se que entre as crianças e adolescentes a porcentagem de aumento seja de 3 a $4 \%$ ao ano (SOCIEDADE BRASILEIRA DE DIABETES, 2019).

O impacto do diagnóstico sobre a família, a criança e ao adolescente envolvem vários aspectos como, sociais, econômicos, afetivos e comportamentais, principalmente pelas complicações que a doença pode gerar. Alguns sentimentos como ansiedade, medo, incertezas podem emergir diante do diagnóstico, principalmente ao se ver diante do desafio de aprender a lidar com os diversos sintomas do agravo, os procedimentos terapêuticos e o desenvolvimento de habilidades para o manejo e controle da doença (MALAQUAIS TSM, et al., 2016).

Para as crianças e adolescentes, o impacto da doença se manifesta por meio de sentimentos de negação, minimização da doença, raiva, frustração pela limitação da doença, sintomas depressivos, culpa e outros (LIMA L, et al., 2017).

A adolescência é caracterizada como um período de transição e este pode ser um fator de risco para a adesão ao tratamento e manejo adequado da doença. Para o pré-adolescente, a vivência adequada com a sua nova condição causada pela doença está relacionada ao desenvolvimento do auto cuidado, ao quanto ele sabe e entende sobre ela, seu cotidiano, vivência escolar, apoio dos amigos, familiares e profissionais que o acompanham (COLLET N, et al., 2018).

Nesse sentido, temos que o cuidado dispensado à pessoa com doença crônica, em especial àquelas na faixa etária infanto-juvenil, deve ser regido e acompanhado pelas Redes de Atenção à Saúde (RAS), visando assistência contínua e integral. O foco deve ser na não fragmentação da assistência, e sim no conjunto de ações que estreitem e melhore as relações entre o usuário e as equipes de saúde, a fim de definir metas e estabelecer plano de cuidados adequados, empoderando famílias e clientes, com foco no autocuidado apoiado (BRASIL, 2014).

Muitas vezes o diagnóstico de um agravo como o DM1 não acontece de forma linear, mas sim de uma maneira repentina, tornando o primeiro atendimento como algo emergencial (CRUZ DSM, et al., 2017), e geralmente realizado pelo Serviço de Atendimento Móvel de Urgência (SAMU) (JESUS YVGS, 2019), para posteriormente ser acompanhado pelas RAS juntamente com o apoio da família (FERNANDEZ HGC, et al., 2019). 
O SAMU faz parte da Rede de Atenção às Urgências (RUE), cuja finalidade é articular e integrar os equipamentos de saúde, além de ampliar e qualificar o acesso humanizado e integral aos usuários em situação de urgência e emergência, de forma ágil e oportuna (BRASIL, 2010).

A solicitação do serviço é realizada por ligação telefônica para a Central de Regulação que identifica a emergência. O médico regulador faz o diagnóstico da situação e inicia o atendimento, com orientações ao paciente ou solicitante sobre as primeiras ações a serem tomadas e solicita o deslocamento de uma ambulância de atendimento ao local, ou então orienta o mesmo a buscar um serviço de saúde (BRASIL, 2006).

Pensando que o acompanhamento do DM1 deve ser realizado de forma contínua e integrada, entre famílias e profissionais de saúde, mas que em muitas situações, o diagnóstico da doença se faz de forma emergencial, a presente investigação tem por objetivo, descrever a configuração familiar e o apoio social de famílias de crianças e adolescentes com suspeita de DM1 atendidos pelo SAMU.

\section{MÉTODOS}

Trata-se de um estudo descritivo, de abordagem qualitativa. A fonte de informação foi um banco de dados secundários do SAMU. A coleta foi realizada por meio de consulta a este banco no segundo semestre de 2015. Esses dados dizem respeito aos atendimentos do Suporte Avançado de Vida do SAMU-192 do noroeste do Paraná, realizados no ano de 2014, registrados no Sistema Estadual de Regulação das Ocorrências, e alimentado pela equipe do SAMU conforme atendimento prestado.

Desses dados foram separados para análise específica atendimentos segundo os seguintes critérios de inclusão: crianças e adolescentes com idade entre 0 e 19 anos na ocasião do atendimento, presença de alteração de glicemia mensurada no momento do atendimento, e informação de contato telefônico. Como critério de exclusão, adotou-se a falha no contato telefônico por três vezes. Ressalta-se que a idade incluída se justifica pela adoção da faixa etária de adolescentes da Organização Mundial da Saúde, a qual abarca até os 19 anos (WHO, 1986).

Os dados coletados foram organizados em uma planilha no Microsoft Exce淄 2016 por faixa etária, sexo, tipo de ocorrência e desfecho dos atendimentos às crianças/adolescentes, fornecendo um total de 100 ocorrências. Deste total foram excluídos os registros que não apresentavam o contato telefônico dos pacientes atendidos/famílias, resultando em 32 ocorrências.

Estas ocorrências foram divididas por alterações na faixa de glicemia, como faixa de glicemia maior ou igual a $300 \mathrm{mg} / \mathrm{dL}$, com 2 ocorrências; de 200 a $299 \mathrm{mg} / \mathrm{dL}$ com 4 ocorrências; de 126 a 199mg/dL com 24 ocorrências. Os casos de hipoglicemia, com valores mensurados de $60 \mathrm{mg} / \mathrm{dL}$ ou menos, corresponderam apenas a duas ocorrências.

Na segunda fase da pesquisa, foi realizado contato telefônico prévio com os participantes considerados elegíveis a partir dos critérios de inclusão, resultando um total de cinco adolescentes. Vinte e seis sujeitos foram excluídos da pesquisa, uma parte por não aceitarem participar do estudo e outra parte pela impossibilidade de acesso aos mesmos após três tentativas de contato telefônico.

Junto àqueles que concordaram em participar da pesquisa, foi realizada entrevista com o responsável juntamente com o adolescente, a partir da obtenção de anuência expressa registrada em Termo de Consentimento Livre e Esclarecido.

Os dados foram coletados por meio de realização de entrevistas utilizando roteiro semiestruturado com a finalidade de obter dados que subsidiassem a construção do genograma e do ecomapa de cada família.

O genograma é definido como uma representação gráfica da composição familiar e dos relacionamentos básicos em, pelo menos, três gerações, elaborada por meio de símbolos. Ele permite, de uma forma rápida e clara, visualizar quais são os membros que constituem a família, tenham eles vínculos consanguíneos ou não, identificando a idade, a ocupação, a profissão e a escolaridade de cada pessoa, além de retratar o lugar ocupado por cada um dentro da estrutura familiar. O ecomapa, por sua vez, é um diagrama das relações 
entre a família e a comunidade e auxilia na avaliação dos apoios disponíveis e a sua utilização pela família. Pode representar a presença ou a ausência de recursos sociais, culturais e econômicos, sendo o retrato de um determinado momento na vida dos membros da família e, portanto, é dinâmico (SOUZA IP, et al., 2016).

Tais ferramentas permitem sistematizar, sintetizar e conferir visibilidade aos modos como se organizam as redes para o cuidado à saúde tecidas por essas pessoas, mostrando quem as compõe, a qualidade de suas relações e os significados produzidos no seu contexto. Auxiliam também na avaliação da composição e organização familiar, sendo uma rica fonte de informação apresentada de forma clara e sucinta (CATANNI NA, et al., 2020).

Deste modo, pretende-se delinear a rede de apoio social e a configuração familiar das crianças/adolescentes atendidas pelo SAMU com alteração glicêmica e suspeita de DM1, de modo a verificar como se configura o atendimento emergencial neste contexto de atenção à saúde, bem como delinear o comportamento desta rede no suporte aos casos eventualmente diagnosticados com este agravo.

A análise dos genogramas e ecomapas, foi realizada de maneira descritiva. Este estudo seguiu todos os preceitos éticos e legais das Resoluções 466/2012 (BRASIL, 2012) e 510/2016 (BRASIL, 2016) do Conselho Nacional de Saúde para pesquisas com seres humanos e foi aprovado pelo Comitê Permanente de Ética de Pesquisa com Seres Humanos da Universidade Estadual de Maringá, sob o parecer nํ⒈375.760/2015.

\section{RESULTADO E DISCUSSÃO}

Participaram do presente estudo um total de cinco adolescentes. Com relação aos resultados obtidos sobre a caracterização dos adolescentes que sofreram o evento de alteração da glicemia, todas eram do sexo feminino, três possuíam 18 anos, uma possuía 17 anos e outra possuía 14 anos no momento da ocorrência. Em relação ao estado civil, as cinco adolescentes eram solteiras. Quanto ao grau de escolaridade, uma adolescente possuía o ensino fundamental incompleto, uma possuía o ensino médio incompleto e três possuíam o ensino médio completo.

O resultado deste estudo está organizado em duas apresentações. A primeira é voltada a uma breve caracterização das famílias; e a segunda, ao delineamento da estrutura interna familiar e de suas relações com outros segmentos da sociedade, por meio da utilização de genograma e ecomapa.

Os diagramas de genograma e ecomapa foram utilizados para melhor visualização e representação dos temas e subtemas que caracterizam a configuração e experiência familiar diante do evento de atendimento pelo SAMU.

\section{Caracterização estrutural da família}

Neste estudo, as famílias foram caracterizadas quanto à sua composição/estrutura, e o apoio social que recebem. Essa caracterização é apresentada aqui, por meio do genograma e ecomapa agrupado. Com a finalidade de preservar o anonimato dos entrevistados e de cada família participante do estudo, as mesmas foram identificadas pelo nome de pássaros da fauna brasileira: Andorinha, Bem-te-vi, Beija Flor, Arara e Tucano.

Família Andorinha: classifica-se como sendo do tipo nuclear, composta por mãe, pai e duas filhas. Reside em casa própria, de alvenaria. A região é assistida por Unidade de Saúde da Família, porém a família não utiliza os serviços da referida unidade, pois a mesma possui plano de saúde.

A entrevistada, representada pela adolescente que vivenciou o evento, está inserida no mercado de trabalho, possui renda própria e ensino médio completo. Cabe destacar que todos os membros da família possuem grau equivalente de instrução. Quanto a religião, foi referida a religião evangélica.

No que tange ao histórico de morbidade, foi referido que o pai do indivíduo índice possui fibromialgia, não havendo diagnóstico de doença crônica não transmissível entre os demais membros da família nuclear. Foi referenciado que a avó paterna faleceu por câncer. A representação da família Andorinha, segue na Figura 1. 
Figura 1 - Genograma e ecomapa da família Andorinha. Maringá-PR, 2015.

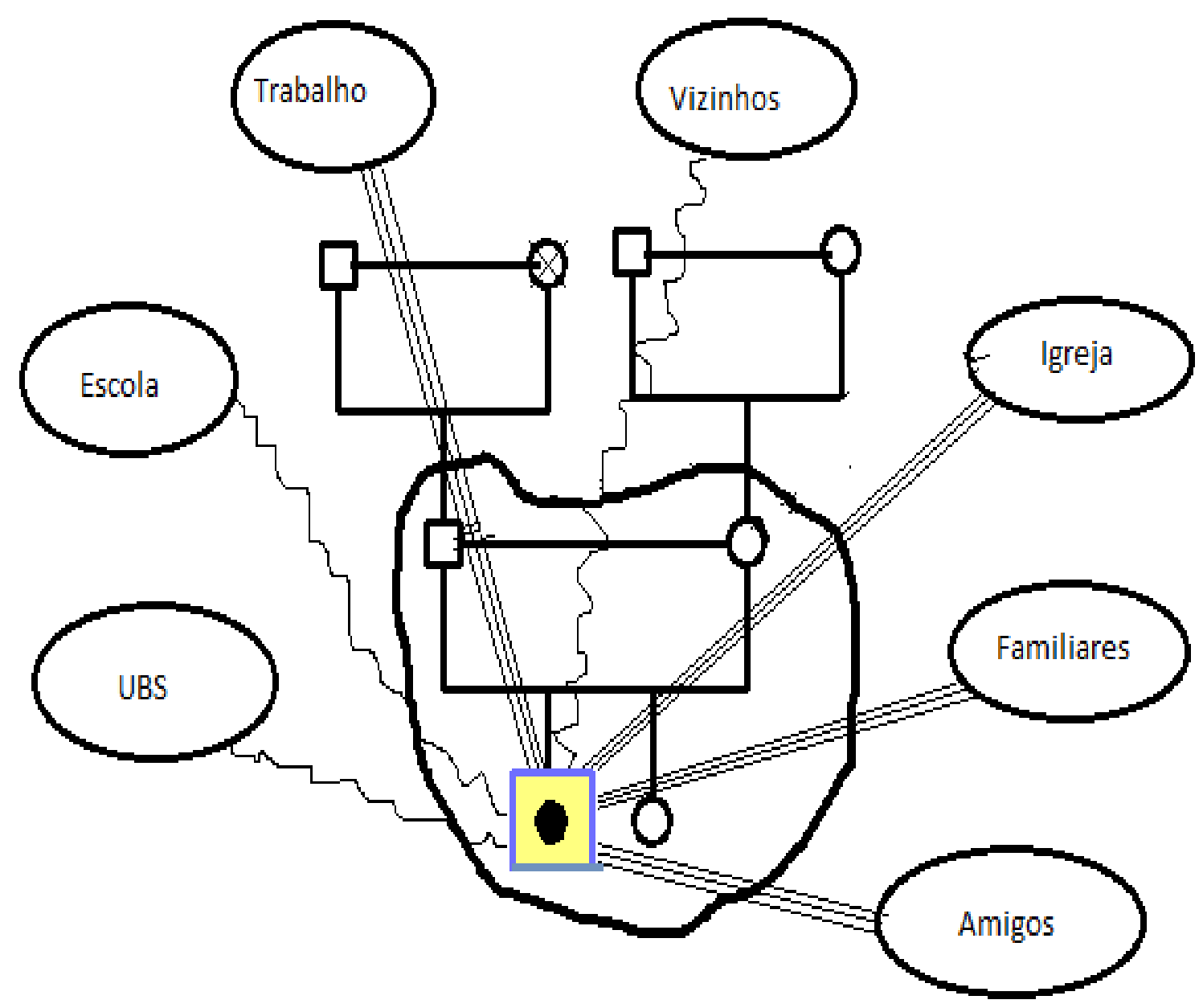

Legenda: Quadrado: pessoa do sexo masculino; Círculo: pessoa do sexo feminino; Círculo com " $x$ ": morte de uma mulher; Linhas horizontais: casamento; Quadrado amarelo com círculo preto: pessoa índice participante da entrevista; Três linhas: vínculos moderados; Linhas onduladas: vínculos negativos.

Fonte: SILVA MGS, et al., 2015.

Com relação ao apoio social, representada pelo ecomapa, foi possível observar que os vínculos moderados da família Andorinha estão estabelecidos com os seguintes segmentos da comunidade: Igreja, Trabalho, Família extensa e amigos. Os vínculos com o serviço de Saúde, Escola e vizinhos foi referido como Frágil/Fraco, principalmente em relação ao indivíduo índice (atendido com alteração glicêmica).

Família Bem-te-vi, do tipo nuclear, formada por pai, mãe, filha e filho. Reside em casa própria, de alvenaria. A família é assistida por Unidade Básica de Saúde, sendo uma área coberta pelos Agentes Comunitários de Saúde (ACS). Contudo, a família não frequenta com regularidade a unidade.

A entrevistada, representada pela adolescente que vivenciou o evento, referiu estar inserida mercado de trabalho, possuindo, portanto, renda própria. Tanto esta, quanto os demais membros da família, apresentam o ensino médio completo. Quanto a religião, foi referida a prática da religião católica.

No que se refere ao histórico de morbidade, foi relatado que nenhum membro da família nuclear apresenta diagnóstico de doenças crônicas não transmissíveis. No entanto, houve relatos com relação à presença de Hipertensão Arterial da avó materna, e de Diabetes mellitus tipo 2, do avô paterno, já falecido. A representação da família Bem-te-vi, segue na Figura 2. 
Figura 2 - Genograma e Ecomapa da família Bem-te-vi. Maringá-PR, 2015.

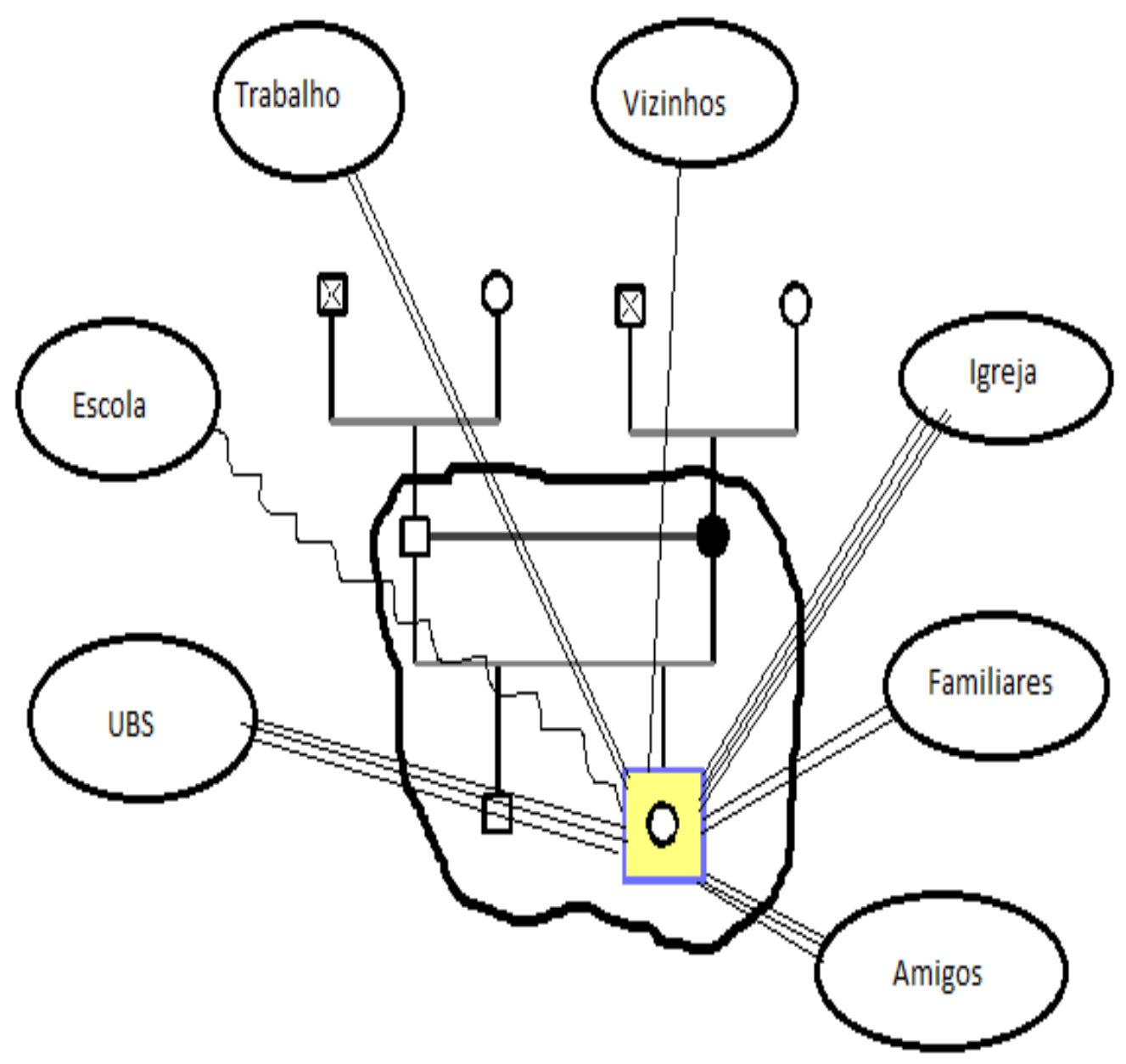

Legenda: Quadrado: pessoa do sexo masculino; Círculo: pessoa do sexo feminino; Quadrado com "x": óbito masculino; Linhas horizontais: casamento; Quadrado amarelo com círculo preto: pessoa índice; Círculo em negrito: participante da entrevista; Duas linhas: vínculos superficiais; Três linhas: vínculos moderados; Quatro linhas: Vínculos fortes; Linhas onduladas: vínculos negativos.

Fonte: SILVA MGS, et al., 2015.

Com relação ao apoio social, representada pelo ecomapa, foi possível observar que o vínculo forte da família Bem-te-vi está estabelecido com o segmento da comunidade "Igreja", já os vínculos moderados foram estabelecidos com o serviço de saúde e amigos. Os vínculos com o Trabalho e com os Familiares foram referidos como superficiais, por parte da entrevistada que também se constitui em indivíduo índice. O vínculo com vizinhos, por sua vez, se mostrou superficial em relação a todos os membros da família Bem-te-vi.

A Família Beija Flor se configura como sendo do tipo nuclear, formada por pai, mãe e filha. Com relação às condições de moradia, a família reside em casa própria de alvenaria. É assistida por uma Unidade Básica de Saúde, sendo a área coberta pelos Agentes Comunitários de Saúde (ACS). A família frequenta com regularidade a unidade.

A entrevistada, representada pela adolescente que vivenciou o acontecimento, está cursando o ensino médio, e a renda familiar é proveniente de seus pais. A família refere não professar qualquer religião. No concernente ao histórico de morbidade da família nuclear, nenhum membro apresenta diagnóstico de doença crônica não transmissível. Foi relatado que apenas o avô paterno encontra-se diagnosticado com câncer. A representação da família Beija Flor, segue na Figura 3. 
Figura 3 - Genograma e Ecomapa da família Beija Flor. Maringá-PR, 2015.

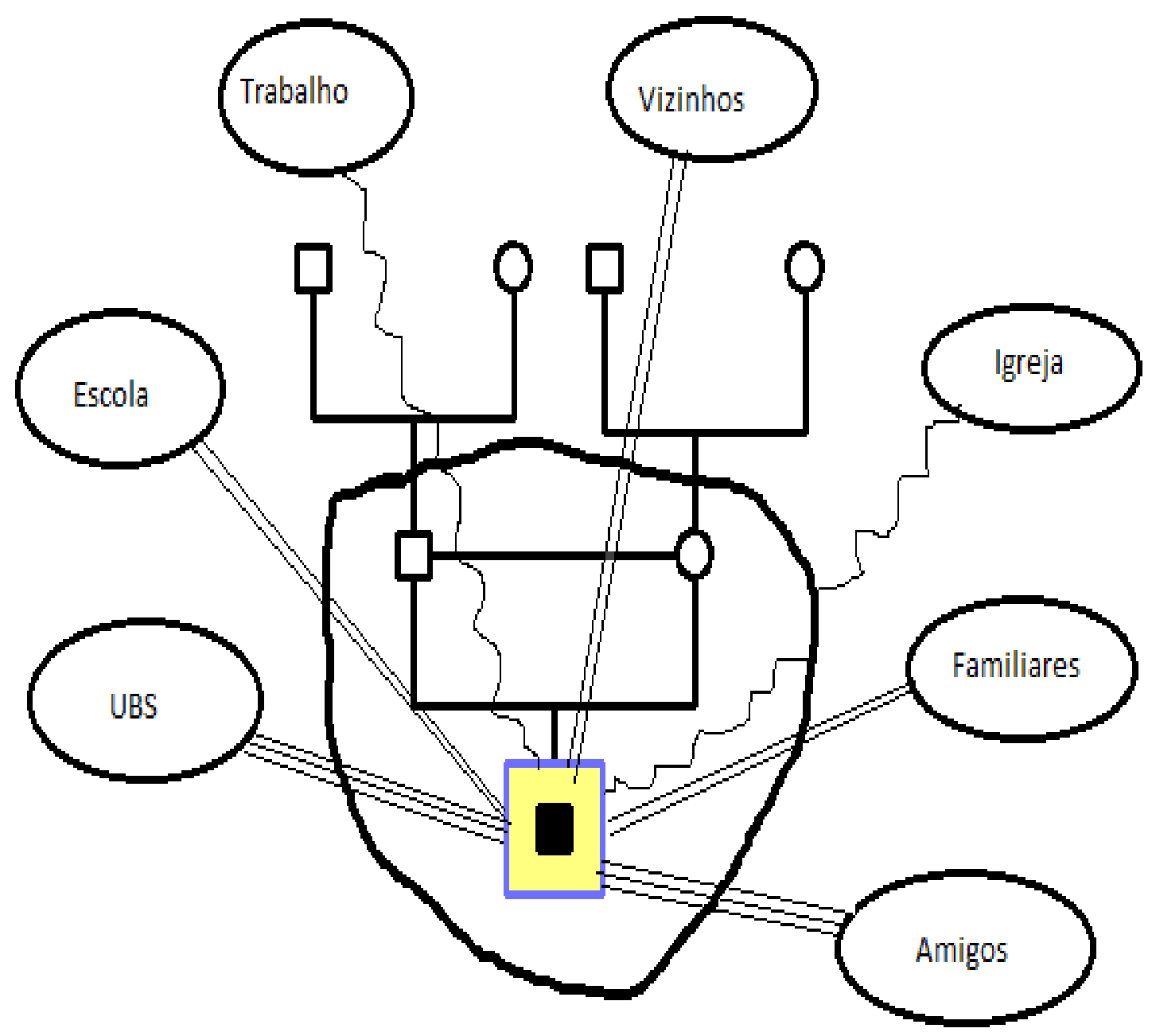

Legenda: Quadrado: pessoa do sexo masculino; Círculo: pessoa do sexo feminino; Linhas horizontais: casamento; Quadrado amarelo com quadrado preto: pessoa índice participante da entrevista; Duas linhas: vínculos superficiais; Três linhas: vínculos moderados; Linhas onduladas: vínculos negativos.

Fonte: SILVA MGS, et al., 2015.

O apoio social representado pelo ecomapa, nos mostra que, em relação à Unidade de Saúde e aos Amigos, os vínculos estão estabelecidos como moderados. Os vínculos com os vizinhos foram definidos como sendo superficiais, e com a Igreja e escola, como vínculos negativos.

A Família Arara, é do tipo nuclear, representada por pai, mãe e filha. Reside em casa própria de alvenaria. A família é assistida por uma Unidade Básica de Saúde, com cobertura por Agentes Comunitários de Saúde (ACS). No entanto, a família não realiza acompanhamento junto a unidade de saúde, preferindo utilizar-se de plano de saúde.

A entrevistada, mais uma vez representada pelo indivíduo índice (adolescente que vivenciou a ocorrência), também encontrava-se trabalhando à época da entrevista, possuindo renda própria e ensino médio completo. Os demais membros da família nuclear possuem o mesmo nível de instrução. Quanto à religião, foi referida a religião evangélica.

No que tange ao histórico de morbidade da família nuclear, nenhum membro desta apresentava diagnóstico de doença crônica não transmissível. A representação da família Arara, segue na Figura 4. 
Figura 4 - Genograma e Ecomapa da família Arara. Maringá-PR, 2015.

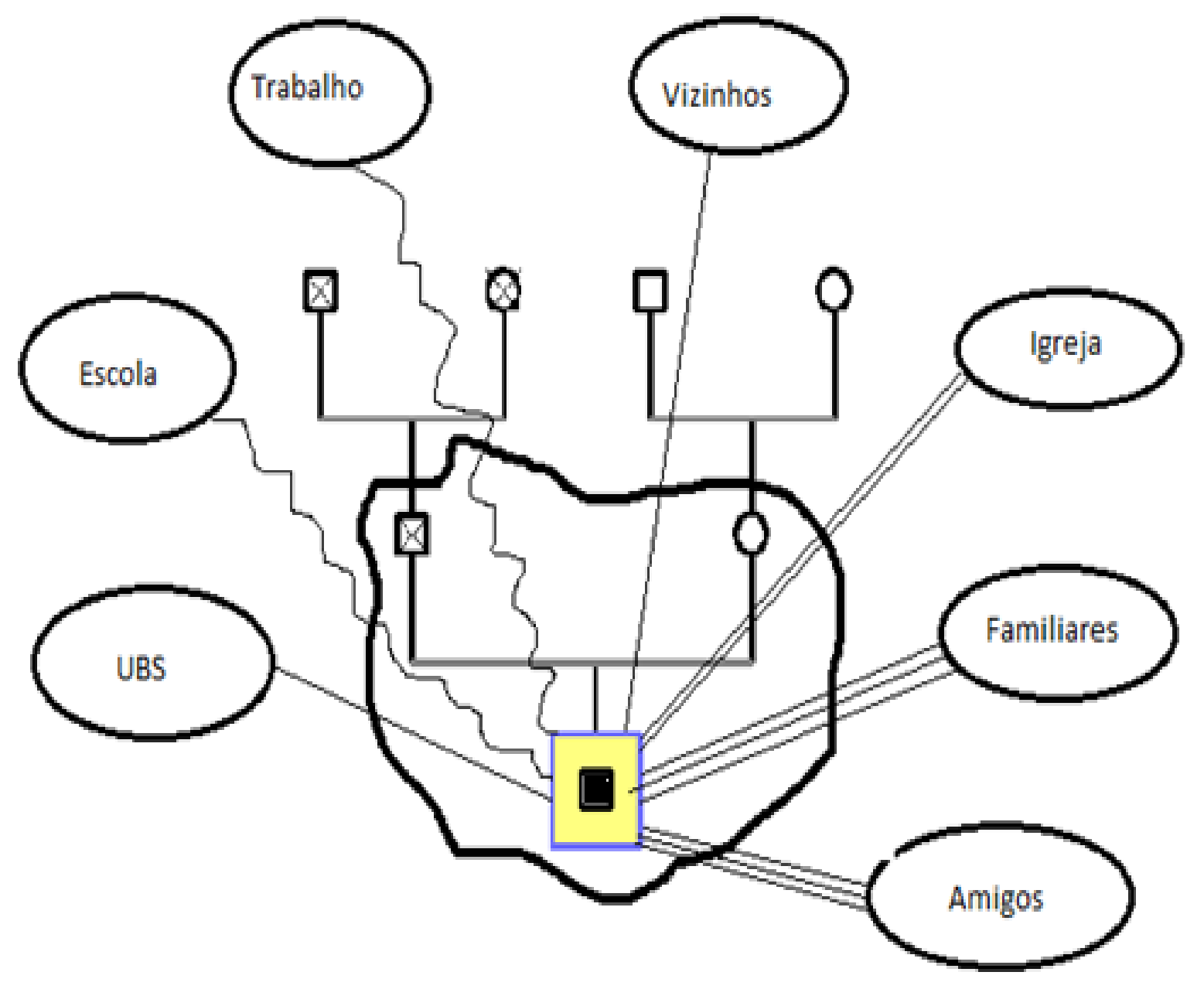

Legenda: Quadrado: pessoa do sexo masculino; Círculo: pessoa do sexo feminino; Quadrado com "x": óbito masculino; Círculo com " $x$ ": morte feminina; Linhas horizontais: casamento; Quadrado amarelo com quadrado preto: pessoa índice participante da entrevista; Uma linha: vínculo muito superficial; Duas linhas: vínculos superficiais; Três linhas: vínculos moderados; Linhas onduladas: vínculos negativos.

Fonte: SILVA MGS, et al., 2015.

Com relação ao apoio social delineado no ecomapa, foi possível observar que os vínculos moderados da Família Arara envolvem os seguintes segmentos da comunidade: Família extensa e Amigos. Vínculos muito superficiais estão estabelecidos com a Unidade de Saúde e com os Vizinhos, e um vínculo superficial com relação à Igreja. Os segmentos da comunidade representados pela Escola e pelo Trabalho, mostram-se vinculados de forma negativa com a família.

A família Tucano, classificada como família do tipo monoparental, formada pela mãe e duas filhas, a família Tucano reside em casa própria de alvenaria, e é atendida pela Equipe de Saúde da Família, sendo visitada pelo Agente Comunitário de Saúde (ACS).

A entrevistada, representada pela adolescente que vivenciou o acontecimento, possui ensino médio completo, assim como os demais membros da família, estando inserida o mercado de trabalho, com renda própria. Todos os membros da família frequentam a igreja. No que diz respeito à morbidade da família Tucano, nenhum membro possui diagnóstico de Doenças Crônicas Não Transmissíveis (DCNT). Foi referido que o avô paterno apresenta diagnóstico de Diabetes mellitus tipo 2. A representação da família Tucano, segue na Figura 5. 
Figura 5 - Genograma e Ecomapa da Família Tucano. Maringá-PR, 2015.

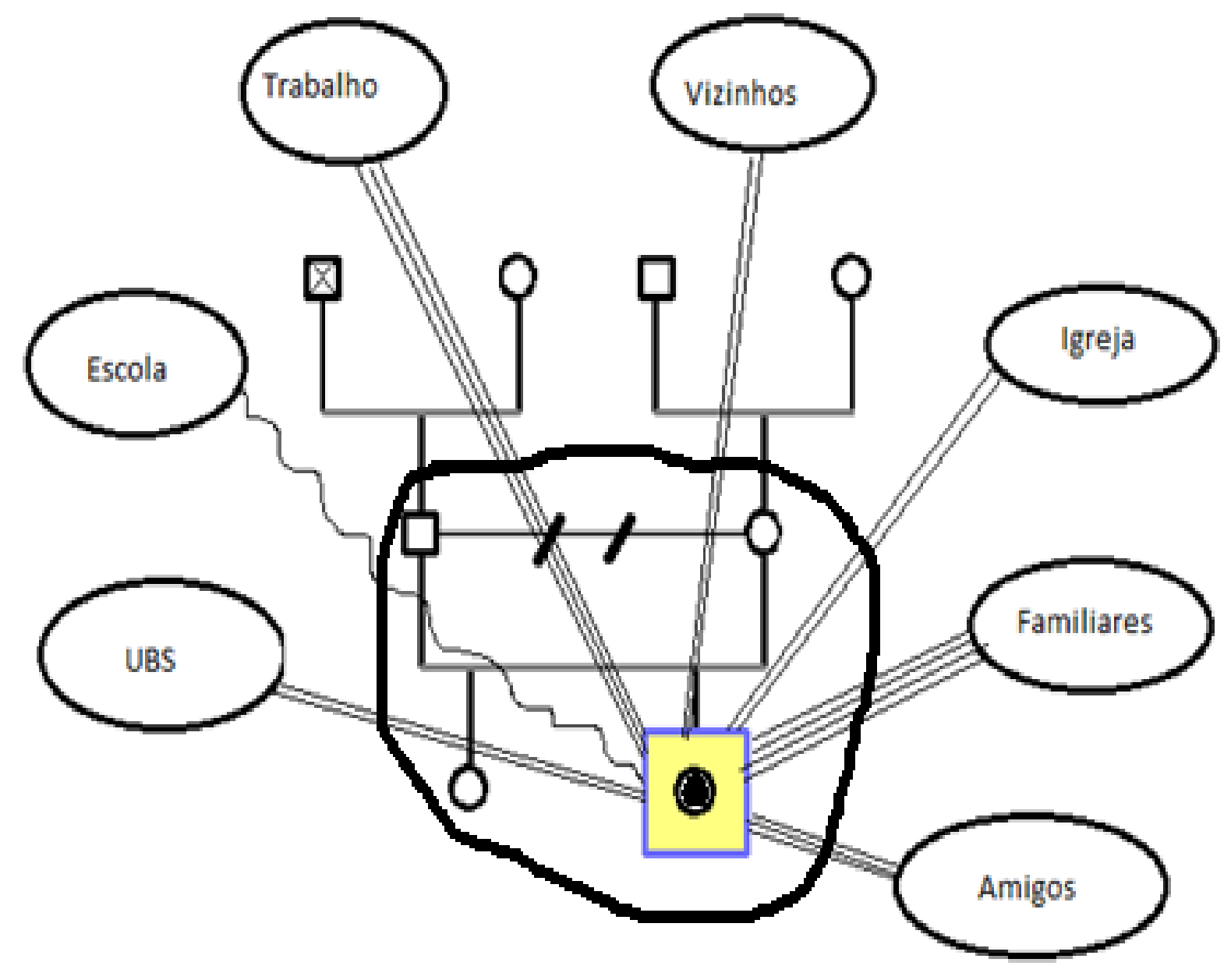

Legenda: Quadrado: pessoa do sexo masculino; Círculo: pessoa do sexo feminino; Quadrado com "x": óbito masculino; Linhas horizontais: casamento; Duas linhas paralelas sobre uma linha horizontal: divórcio; Quadrado amarelo com círculo preto: pessoa índice participante da entrevista; Duas linhas: vínculos superficiais; Três linhas: vínculos moderados; Quatro linhas: vínculos fortes; Linhas onduladas: vínculos negativos.

Fonte: SILVA MGS, et al., 2015.

Com relação ao apoio social, configurado pelo ecomapa, foi possível observar que os vínculos moderados da família Tucano estão estabelecidos com os seguintes segmentos da comunidade: Amigos e Trabalho. Os vínculos com o serviço de Saúde, vizinhos e igreja foram referidos como superficiais, principalmente em relação ao indivíduo índice. O vínculo com a Família extensa foi considerado forte.

A partir da descrição dos resultados foi possível verificar que nenhuma das participantes teve diagnóstico firmado de Diabetes mellitus por ocasião ou após o atendimento realizado pelo Serviço Móvel de Urgência (SAMU). Os motivos que ocasionaram o chamado do SAMU foram: lipotímia ou desmaio sem causa específica e, em um dos casos, ingestão de veneno para formigas. Em relação ao local onde teve lugar o incidente, um ocorreu no ambiente de trabalho, outro na rua, e três em casa. Dois casos dos atendimentos apresentaram hipoglicemia e três hiperglicemias.

Os sintomas clássicos do DM1 incluem a polidipsia e a perda de peso progressiva entre 2 e 6 semanas. Em pacientes que evoluem com a complicação mais grave do DM1, como a cetoacidose diabética (CAD) podem aparecer em maior frequência: náuseas, vômitos, dor abdominal, taquipneia ou dispneia e alteraões do nível de consciência (International Society for Pediatric and Adolescent Diabetes (ISPAD), 2014). 
Percebe-se que a maioria das participantes possuía bom nível socioeconômico, o que favorecia a utilização de plano de saúde privado, e refletia-se na baixa utilização dos serviços públicos de saúde. Esta também pode ser a causa para a configuração de vínculos mais superficiais destes núcleos familiares com as Unidades Básicas de Saúde, conforme demonstraram os ecomapas das famílias.

A interdependência entre os sistemas públicos e privados de saúde, já existe há mais de 30 anos, no qual apesar de o país possuir um sistema de saúde gratuito, algumas pessoas optam pelo setor privado. A decisão e utilização dos serviços de saúde é um comportamento complexo que envolve variáveis sociais, econômicas e demográficas (VIACANA F, et al., 2018).

Deste modo, e não obstante o fato de todas as famílias serem visitadas pela Equipe de Saúde da Família, e, portanto, terem conhecimento acerca dos serviços ofertados pela atenção básica, a baixa utilização destes serviços e os vínculos superficiais apresentados, podem ser decorrentes das concepções destas famílias de que a atenção dispensada via plano de saúde pudesse ter qualidade superior ou se dar de forma mais ágil (ZATTA FN, et al., 2019).

Esta busca pela rapidez e prontidão de atendimento também pode explicar a opção pelo SAMU, para o atendimento do incidente apresentado pelas adolescentes deste estudo. Sabe-se que não são raras as vezes em que o serviço é acionado por motivos que não se enquadram na condição de urgência/emergência. Os impactos desta subutilização de serviço especializado são diversos, e incluem, entre outros, o desvio deste transporte de um local onde a sua necessidade era real (ABREU KP, et al., 2012; ALMEIDA RB e ALVARES ACM, 2019; SOUZA LC, et al., 2020).

As justificativas para este comportamento de utilização e busca pelo SAMU estão atreladas à concepção de que este serviço se constitui em porta de entrada rápida e garantida aos serviços de saúde (ORTIGA AMB, et al., 2016).

No caso das entrevistadas, as mesmas relataram desconhecer o fato deste serviço não se constituir em porta de entrada indicada para o tipo de evento apresentado, bem como referiram não saber que poderiam ter optado por buscar a Unidade de Saúde mais próxima da sua residência para o atendimento, ou mesmo, para o encaminhamento posterior ao serviço de referência.

Com relação à presença de DCNT na família, a totalidade referiu a inexistência destes diagnósticos entre os membros da família nuclear, e a existência de antecedentes mórbidos familiares envolvendo a família extensa (avós). As DCNT mais relatadas foram a hipertensão, o DM tipo 2 e o câncer, todos com início na idade adulta ou terceira idade.

Tal investigação é de extrema importância, na medida em que o DM1 está associado a características genéticas e fatores hereditários que são condicionantes para a predisposição à doença (DIB AS, et al., 2008). Além disso, sabe-se que as crianças que possuem histórico familiar de DM tem um risco aumentado de vir a desenvolver diabetes em relação à população em geral e que fatores ambientais, como infecções, podem contribuir para desencadear uma resposta imune contra os antígenos das células beta, causando sua destruição (POCIOT F e LERNMARK A, 2016; JERRAM ST e LESLIE RD, 2017; CHIANG JL, et al., 2018).

A idade inferior a cincos anos, o baixo nível socioeconômico, o diagnóstico tardio do DM1 e a residência em países com baixa prevalência da doença, são fatores de risco para o aparecimento de complicações graves, dentre elas, a cetoacidose diabética (CAD). Supõe-se que uma prevalência mais alta do DM1 levaria a uma maior conscientização dos familiares e profissionais de saúde, visando a identificação de sintomas prévios que antecipariam o diagnóstico e o tratamento. Ao mesmo tempo, o histórico familiar de DM1 pode ser considerado como um fator de proteção para a CAD no momento do diagnóstico, visto que a percepção de alterações na criança e no adolescente seriam facilmente identificadas (SOUZA LC, et al., 2020).

A frequência da cetoacidose diabética no momento do diagnóstico do DM1, que é uma das complicações mais graves da doença varia de uma região para outra. Alguns estudos brasileiros trazem uma incidência que varia de 42 a $67 \%$ e esses dados elevados podem estar relacionados a falhas no atendimento médico a pacientes com sintomas sugestivos de DM1 (SOUZA LCVF, et al., 2020). 


\section{CONCLUSÃO}

Descobriu-se que a maioria das famílias é nuclear (pai, mãe e filhos), possuem casa própria, referem atendimento pela equipe da atenção básica de saúde, porém possuem plano de saúde privado. A maioria dos participantes desconhecia o real motivo de urgência que deve ser encaminhado ao SAMU e de que poderiam utilizar a atenção primária para tal atendimento. Os vínculos de apoio mais citados foram a família extensa, igreja, trabalho, vizinhos e amigos. Nenhum dos adolescentes teve a CAD como fator de diagnóstico da doença.

\section{REFERÊNCIAS}

1. ABREU KP, et al. Percepções de urgência para usuários e motivos de utilização do serviço de atendimento préhospitalar móvel. Rev Gaúcha Enferm, 2012; 33(2):146-152.

2. ALMEIDA RB, ÁLVARES ACM. Assistência de enfermagem no serviço móvel de urgência (SAMU): revisão de literatura. Rev Inic Cient Ext, 2019; 2(4):196-207.

3. BRASIL. Ministério da Saúde. Gabinete do Ministro. Resolução no 466, de 12 de dezembro de 2012. Aprova diretrizes e normas regulamentadoras de pesquisas envolvendo seres humanos. Diário Oficial da União, Brasília-DF, 12 dez. 2012.

4. BRASIL. Ministério da Saúde. Gabinete do Ministro. Resolução no 510, de 07 de abril de 2016. Dispõem sobre Procedimentos Metodológicos Característicos das Áreas de Ciências Humanas e Sociais. Diário Oficial da União, Brasília-DF, 24 mai. 2016.

5. BRASIL. Ministério da Saúde. Secretaria de Atenção à Saúde. Departamento de Atenção Especializada. Regulação médica das urgências / Ministério da Saúde, Secretaria de Atenção à Saúde, Departamento de Atenção Especializada. Brasília: Editora do Ministério da Saúde, 2006.

6. BRASIL. Ministério da Saúde; Secretaria de Atenção à Saúde, Departamento de Atenção Básica. Estratégias para o cuidado da pessoa com doença crônica. Brasília: MS; 2014. (Cadernos de Atenção Básica, 35)

7. BRASIL Portaria $n^{\circ} 4.279$, de 30 de dezembro de 2010. Estabelece diretrizes para a organização da Rede de Atenção à Saúde no âmbito do Sistema Único de Saúde [portaria na internet]. Diário Oficial da União 30 dez 2010.

8. CATANNI NA, et al. Family treating a member with mental disorders: genogram and ecomap. Rev. Enferm. UFSM, 2020; 10(6): 1-18.

9. COLLET N, et al. Autocuidado apoiado no manejo da Diabetes tipo 1 durante a transição da infância para adolescência. Rev Esc Enferm. USP, 2018; 52.

10. CHIANG JL, et al. Type 1 Diabetes in Children and Adolescents: A Position Statement by the American Diabetes Association. Diabetes Care, 2018; 41(9):2026-44.

11. CRUZ DSM, et al. Experiencias de madres de niños diabéticos. Esc. Anna Nery, 2017; 21(1): 1-12.

12. DIB AS, et al. Diabetes melito tipo 1: pesquisa à clínica. Arq Bras Endocrinol Metab, 2008; 52(2): 143-145.

13. FERNANDEZ HGC, et al. Making decisions on health care for children / adolescents with complex chronic conditions: a review of the literature. Ciência \& Saúde Coletiva,2019; 24(6):2279-2292.

14. HERMES TSV, et al. Diabetic child of type 1 and family life: repercussions in the management of the disease. Saúde Debate, 2018; 42(119): 927-939.

15. JERRAM ST, LESLIE RD. The Genetic Architecture of Type 1 Diabetes. Genes, 2017; 8(209): 1-8.

16. JESUS, YVGS. Perfil das ocorrências do Serviço de Atendimento Móvel de Urgência - SAMU 192 em Aracaju. Trabalho de Conclusão de Curso (Graduação em Medicina) - Universidade Federal de Sergipe, Lagarto, $2019 ; 40$ p.

17. LIMA, L, et al. How chronic disease affects children's views on being ill and healthy: a comparative study. Scandinavian Journal of Caring Sciences, 2017; 31(4): 922-929.

18. MALAQUIAS TSM, et al. A criança e o adolescente com diabetes mellitus tipo 1: desdobrar do cuidado familiar. Cogitare Enferm, 2016; 21(1): 01-07

19. ORTIGA AMB, et al. Evaluation of the Mobile Emergency Care Service in Santa Catarina State, Brazil. Cad. Saúde Pública, 2016; 32(12): e00176714

20. POCIOT F, LERNMARK A. Genetic risk factors for type 1 diabetes. The Lancet, 2016; 387(10035), 2331-2339.

21. SOCIEDADE BRASILEIRA DE DIABETES (SBD). Diretrizes da Sociedade Brasileira de Diabetes (2019-2020). São Paulo: Clannad, 2019; 491p.

22. SOUZA IP, et al. Genograma e ecomapa como ferramentas para compreensão do cuidado familiar no adoecimento crônico de jovem. Texto contexto, 2016; 25(4): e1530015.

23. SOUZA LC, et al. Fatores associados ao uso não urgente de unidades de pronto atendimento: uma abordagem multinível. Cad Saúde Colet, 2020; 28(1):56-65.

24. SOUZA LCVF, et al. Cetoacidose diabética como apresentação inicial de diabetes tipo 1 em crianças e adolescentes: estudo epidemiológico no sul do Brasil Rev. paul. Pediatr, 2020; 38.

25. VIACANA F, et al. SUS: oferta, acesso e utilização de serviços de saúde nos últimos 30 anos. Ciência \& Saúde Coletiva,2018; 23(6):1751-1762.

26. ZATTA FN, et al. Application of the Analytic Hierarchy Process in the choice of health insurance. Research, Society and Development, 2019; 8(1): e1881532.

27. WHO, World Health Organization. Young People's Health - a Challenge for Society. Report of a WHO Study Group on Young People and Health for All. Technical Report Series 731. Geneva: WHO, 1986. 